\title{
Population genetic estimation of the loss of genetic diversity during horizontal transmission of HIV-I
}

\author{
Charles TT Edwards*1, Edward C Holmes ${ }^{2}$, Daniel J Wilson ${ }^{3}$, \\ Raphael P Viscidi ${ }^{4}$, Elaine J Abrams ${ }^{5}$, Rodney E Phillips ${ }^{1}$ and
} Alexei J Drummond 6,7

\begin{abstract}
Address: ${ }^{1}$ Nuffield Department of Clinical Medicine, University of Oxford, The Peter Medawar Building for Pathogen Research, South Parks Road, Oxford, OX1 3SY, UK, ${ }^{2}$ Department of Biology, The Pennsylvania State University, University Park, PA 16802, USA, ${ }^{3}$ Department of Statistics, University of Oxford, The Peter Medawar Building for Pathogen Research, South Parks Road, Oxford, OX1 3SY, UK, ${ }^{4}$ Department of Pediatrics, The Johns Hopkins Hospital, Baltimore, MD 21287, USA, ${ }^{5}$ Department of Pediatrics, Columbia University College of Physicians and Surgeons and Harlem Hospital Center, NY, USA, ${ }^{6}$ Department of Zoology, University of Oxford, South Parks Road, Oxford, OX1 3PS, UK and ${ }^{7}$ Department of Computer Science, University of Auckland, Private Bag 92019, New Zealand
\end{abstract}

Email: Charles TT Edwards* - cedwards@maths.uct.ac.za; Edward C Holmes - ech15@psu.edu; Daniel J Wilson - daniel.wilson@stats.ox.ac.uk; Raphael P Viscidi - rviscid1@jhmi.edu; Elaine J Abrams - eja1@columbia.edu; Rodney E Phillips - rodney.phillips@ndm.ox.ac.uk; Alexei J Drummond - alexei@cs.auckland.ac.nz

* Corresponding author

Published: 23 March 2006

BMC Evolutionary Biology2006, 6:28 doi:10.1 186/147/-2148-6-28

This article is available from: http://www.biomedcentral.com/I47I-2/48/6/28

(c) 2006Edwards et al; licensee BioMed Central Ltd.

This is an Open Access article distributed under the terms of the Creative Commons Attribution License (http://creativecommons.org/licenses/by/2.0), which permits unrestricted use, distribution, and reproduction in any medium, provided the original work is properly cited.
Received: 04 October 2005

Accepted: 23 March 2006

\begin{abstract}
Background: Genetic diversity of the human immunodeficiency virus type I (HIV-I) population within an individual is lost during transmission to a new host. The demography of transmission is an important determinant of evolutionary dynamics, particularly the relative impact of natural selection and genetic drift immediately following HIV-I infection. Despite this, the magnitude of this population bottleneck is unclear.
\end{abstract}

Results: We use coalescent methods to quantify the bottleneck in a single case of homosexual transmission and find that over $99 \%$ of the env and gag diversity present in the donor is lost. This was consistent with the diversity present at seroconversion in nine other horizontally infected individuals. Furthermore, we estimated viral diversity at birth in 27 infants infected through vertical transmission and found there to be no difference between the two modes of transmission.

Conclusion: Assuming the bottleneck at transmission is selectively neutral, such a severe reduction in genetic diversity has important implications for adaptation in HIV-I, since beneficial mutations have a reduced chance of transmission.

\section{Background}

The size of the inoculum that initiates infection in HIV-1 is unknown, although the loss of diversity is thought to be substantial following both horizontal [1-7] and vertical $[8,9]$ transmission. If the bottleneck is selectively neutral, genetic drift will occur because only a small number of variants are chosen at random from the population to propagate the new infection. The smaller the amount of genetic diversity transmitted the greater the magnitude of drift, lowering the probability that adaptive changes that emerge within hosts will survive transmission. 
In RNA viruses with a high deleterious mutation rate the majority of variants exhibit a replicative capacity lower than the mean [10-12]. Because the fittest variants may only be present at a low frequency, they are susceptible to random loss. Hence when genetic drift is strong, deleterious mutations may accumulate, leading to an irreversible decline in population fitness [13]. Although the high rate of recombination in HIV-1 in vivo [14-16] has the potential to rescue debilitated haplotypes [13], if a new infection is initiated by only one or a few viral particles, and if these are chosen at random from the parent population, then the transmission of HIV-1 will likely incur a substan- tial reduction in fitness [17-21]. As the inoculum size increases, potential fitness losses are rapidly reduced $[22,23]$.

Conversely, natural selection may lower the susceptibility of HIV-1 to reductions of fitness associated with transmission. In acutely infected HIV-1 patients, the usually diverse envelope $V 3$ region is more homogeneous than gag p17, whereas in chronic infection the opposite is true $[6,7]$. Positive selection operating on envelope during transmission has been invoked as an explanation [6,7]. If selection operates to influence which variants are trans- (a) env V1-V4

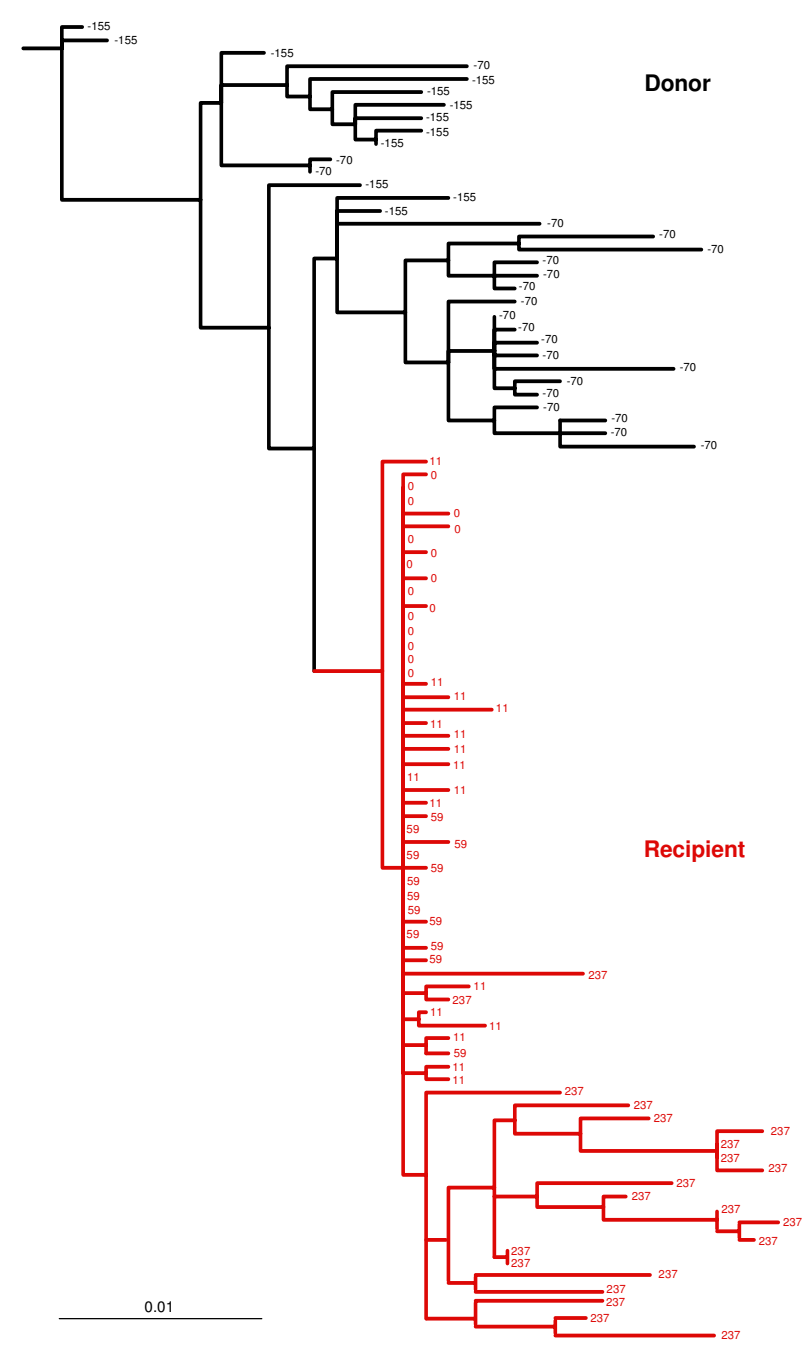

(b) gag p24

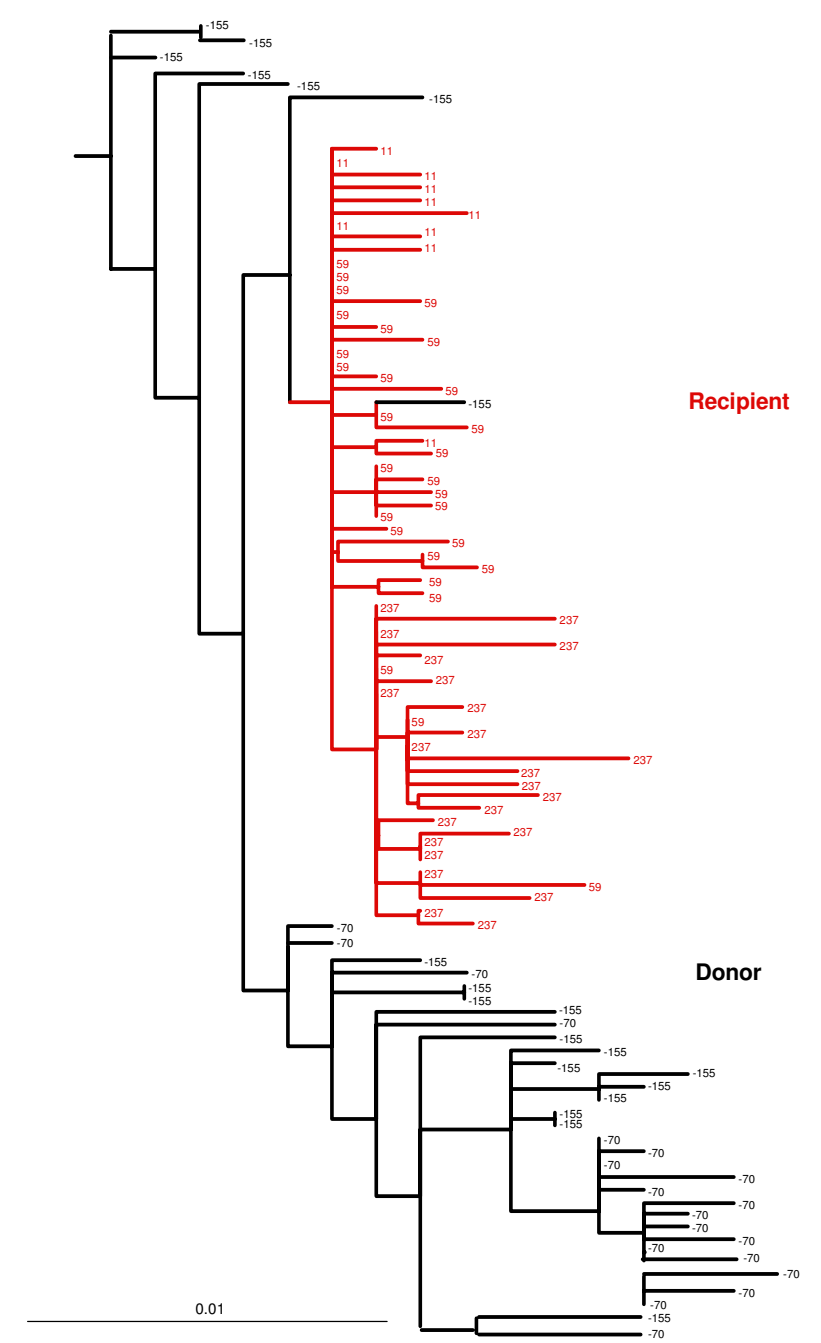

\section{Figure I}

Phylogenetic relationship of (a) env VI-V4 and (b) gag p24 sequences. Maximum likelihood phylogenies depicting the relationship between sequences from donor and recipient, illustrating the reduction in genetic diversity at transmission. Horizontal branch lengths are drawn on a scale of nucleotide changes per site. Branches leading to recipient sequences are highlighted in red, with the day of sample collection relative to the first recipient sample (day 0 ) shown for each sequence. 
Table I: Fit of demographic models

\begin{tabular}{|c|c|c|c|c|}
\hline \multicolumn{4}{|c|}{ Demographic Model } & \multirow{2}{*}{$\begin{array}{c}\text { Coalescent } \\
\text { ESSd }\end{array}$} \\
\hline Recipient & Donor & $\ln L k^{\mathrm{b}}$ & $\mathrm{AICc}$ & \\
\hline \multicolumn{5}{|l|}{ env VI-V4 } \\
\hline Constant & $-\mathrm{a}$ & $-4 \mid 55.385$ & 8310.77 & 523.85 \\
\hline Constant & Constant & $-4 \mid 44.993$ & 8291.99 & 419.77 \\
\hline Exponential & Constant & -4103.717 & 8211.43 & 643.10 \\
\hline Logistic & Constant & -4090.154 & 8186.31 & 126.43 \\
\hline \multicolumn{5}{|l|}{ gag p24 } \\
\hline Constant & $-\mathrm{a}$ & -3118.180 & 6236.36 & 483.84 \\
\hline Constant & Constant & -3121.900 & 6245.80 & 440.34 \\
\hline Exponential & Constant & -3116.760 & 6237.52 & 378.33 \\
\hline Logistic & Constant & -3089.852 & 6185.70 & 202.69 \\
\hline
\end{tabular}

aPopulation size in Recipient constrained to be the same as that in Donor

bNatural logarithm of the likelihood obtained from fitting the demographic model to the data

cAkaike Information Criteria

dEffective Sample Size (number of independent coalescent genealogies sampled from the posterior distribution)

mitted then it will also prevent the fixation of deleterious mutations.

Herein we estimate, using population genetic techniques, the proportion of genetic diversity that survives transmission in a single homosexual transmitter pair, with samples available before and after the transmission event. The demographic history of the virus population in both donor and recipient was reconstructed using coalescent methodology, allowing quantification of the diversity present close to the time of infection. The coalescent was implemented within a Bayesian framework, which enabled co-estimation of substitution and demographic parameters using serially sampled sequences [24-26].

Through a comparison of different regions of the genome (namely env V1-V4 and gag p24) we also investigate whether selection is likely to be acting during HIV-1 transmission. Finally, we generalise our result by estimating the diversity present close to the time of infection in nine homosexual seroconverters for which donor sequences were unavailable, and compare horizontal and vertical modes of transmission using 27 infants infected at birth.

\section{Results}

To directly visualise the change in genetic diversity during horizontal HIV-1 transmission between the donor-recipient pair studied, we first inferred the phylogenetic relationships among their HIV-1 sequences using maximum likelihood methods. The phylogenies for env V1-V4 and gag p 24 depicted in Figure 1 show that branch lengths are substantially shortened immediately after transmission, illustrating that a significant reduction in diversity has occurred.
To investigate the demographics of viral transmission in this transmitter pair more closely, four coalescent models were fitted to the sequence data. Crucially, samples were available both before and after the transmission event allowing distinct demographic functions for donor and recipient HIV-1 populations (Equations 1 to 5), with the time of transition between them estimated from the data [26]. In addition to a null model that constrained the effective population size in donor $\left(N_{D}\right)$ and recipient $\left(N_{R}\right)$ to be identical (so that there is no bottleneck at transmission), models with constant, exponential and logistic demographic functions for the recipient population were fitted. In all cases the donor population size was assumed to be constant.

The relative Bayesian posterior scores for each demographic model are listed in Table 1. For both env V1-V4 and $g a g$ p 24 , the model with the lowest AIC (the preferred model) fits a constant population size in the donor and logistic growth in the recipient (Equations 4 and 5). The null hypothesis that there has been no change in population size at transmission was therefore rejected. Using the estimated model parameters we reconstructed the demographic profiles of genetic diversity $(N \tau$, the product of the effective population size and generation length in days [27]) against time for each gene (Figure 2).

To further test the extent of the transmission bottleneck, the demographic history of the population was reconstructed using the Bayesian skyline plot [see Methods, [28]]. The results for $e n v V 1-V 4$ and gag p24 are shown in Figure 2. In both cases there is a good fit between the demographic profiles estimated using the two different methods. Noticeably, the timing of the transmission bot- 
(a) env V1-V4

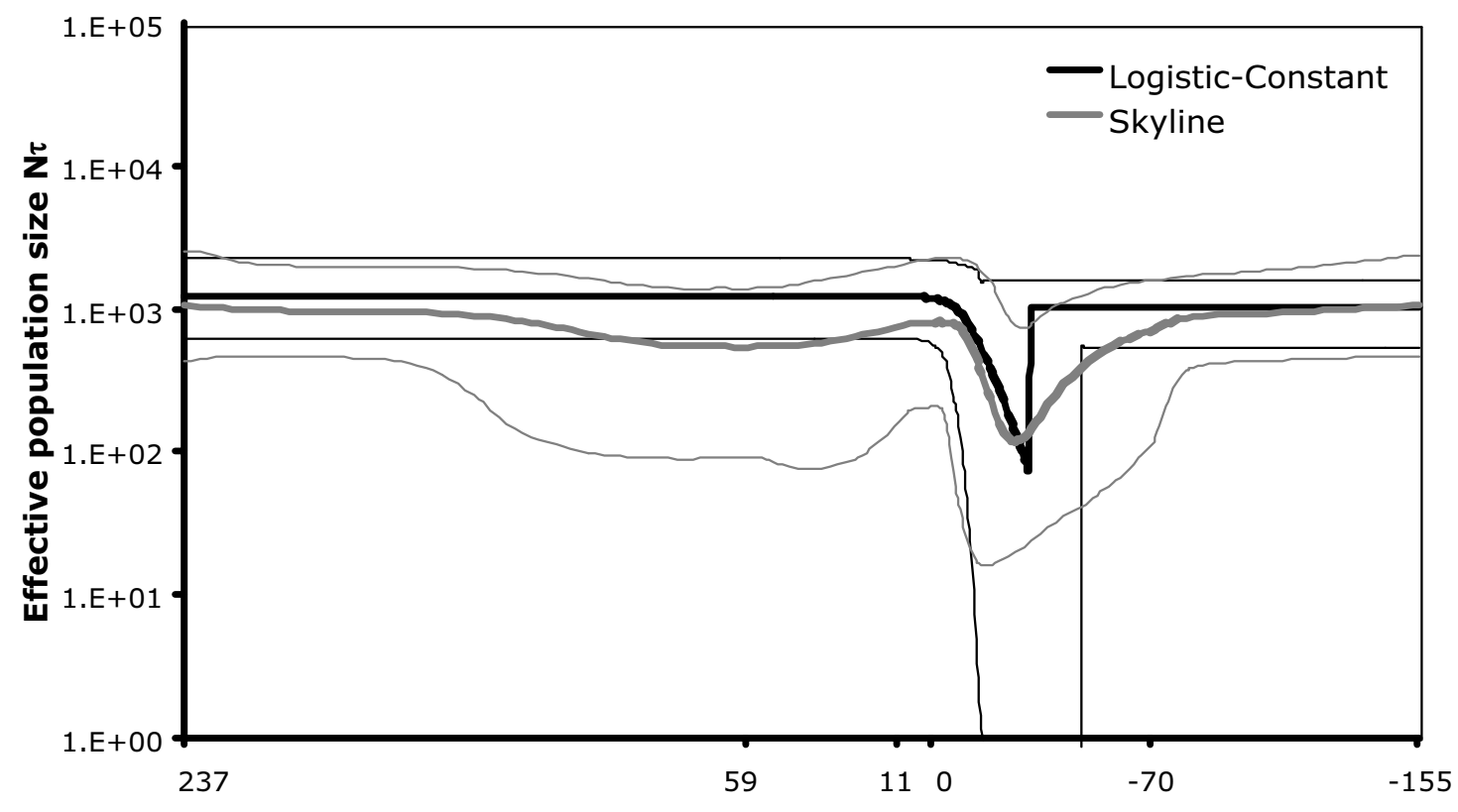

(b) gag p24

Time in days

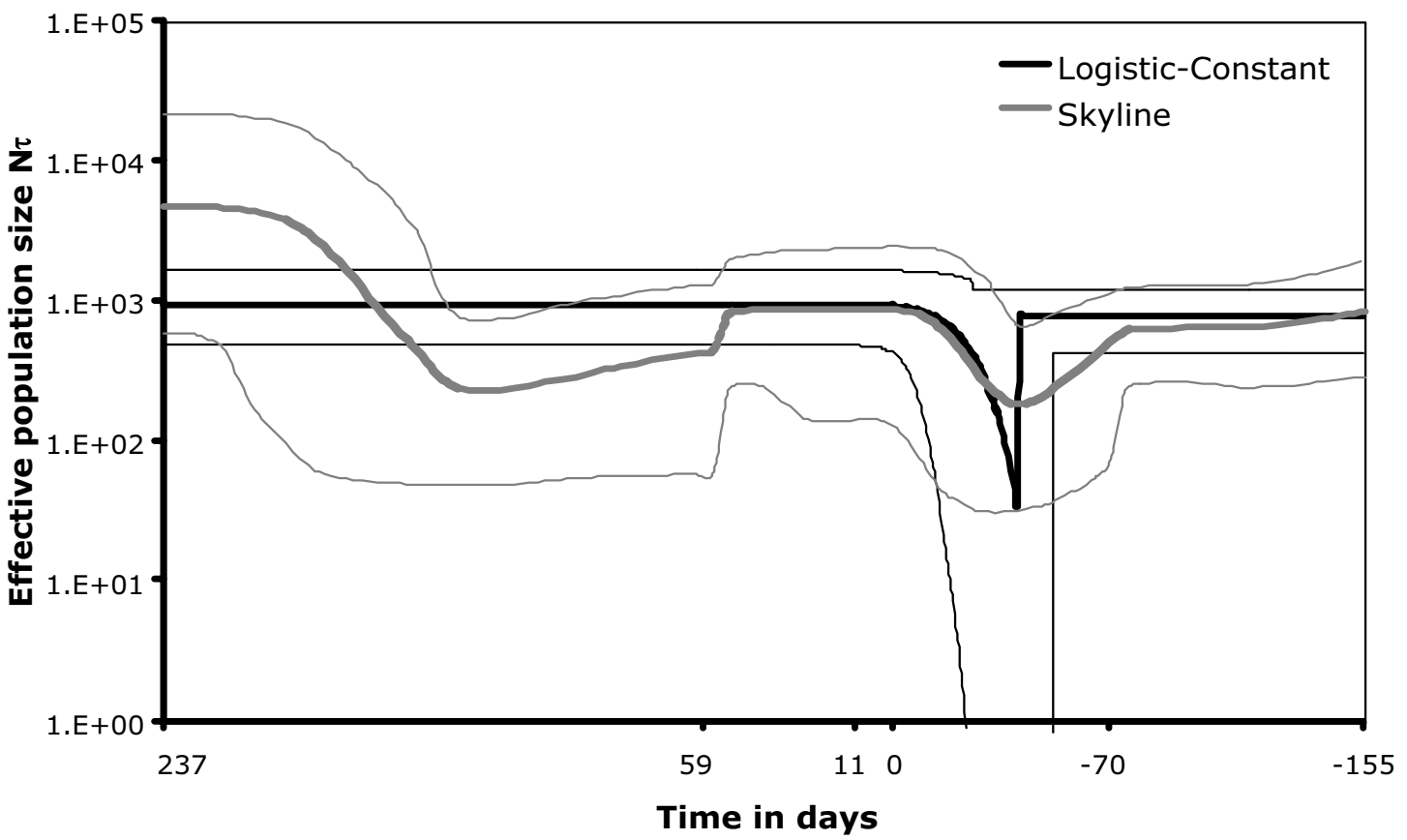

Figure 2

Reconstructed demographic profiles for (a) env VI-V4 and (b) gag p24. Estimates of $N \tau$ are shown on a log scale against time backwards since the most recent sample. Only days on which sequences were sampled are shown, measured relative to the first recipient sample (day 0 ). Mean estimates of $N \tau$ obtained from the best fit Logistic-Constant demographic model and the Bayesian skyline plot are shown with their HPD confidence bounds. 
Table 2: Parameter estimates used to calculate the percentage diversity that survived transmission

\begin{tabular}{|c|c|c|c|c|}
\hline Parameter & Mean ${ }^{a}$ & HPD $^{\mathrm{b}}$ Lower & HPD Upper & ESSc \\
\hline \multicolumn{5}{|l|}{ env VI-V4 } \\
\hline$N_{R} \tau$ & 1216.7 & 534.4 & 2033.9 & 1338.94 \\
\hline$N_{D} \tau$ & 1014.0 & 541.2 & 1538.0 & 955.71 \\
\hline$t_{\text {trans }}{ }^{d}$ & 30.9 & 15.2 & 46.9 & 174.57 \\
\hline$N_{R} \tau\left(t_{\text {trans }}\right)$ & 1.6 & 1.0 & 3.1 & 2456.87 \\
\hline$\delta$ & 0.17 & 0.06 & 0.35 & 2043.08 \\
\hline \multicolumn{5}{|l|}{ gag p24 } \\
\hline$N_{R} \tau$ & 926.6 & 419.9 & 1512.6 & | 454.3 | \\
\hline$N_{D} \tau$ & 770.7 & 413.5 & 1184.7 & 1356.90 \\
\hline$t_{\text {trans }} \mathrm{d}$ & 42.4 & 27.5 & 53.0 & 274.51 \\
\hline$N_{R} \tau\left(t_{\text {trans }}\right)$ & 2.0 & 1.0 & 4.5 & 3532.69 \\
\hline$\delta$ & 0.29 & 0.07 & 0.67 & 3310.56 \\
\hline
\end{tabular}

a Mean of the marginal posterior probability distribution of parameter values

bHighest Posterior Density encompassing $95 \%$ of the marginal posterior distribution of parameter values

cEffective Sample Size (number of independent samples taken from the posterior distribution of values for a particular parameter)

dEstimated time of transmission in days prior to the day of the fist recipient sample (day 0 )

tleneck is the same, and evidence for a bottleneck is readily apparent under both models.

The Bayesian skyline plot also justifies our use of the logistic-constant demographic model to estimate the diversity that survives during horizontal transmission of HIV-1. Using the logistic growth model (Equations 4 and 5) we were able to calculate diversity in the recipient $N_{R} \tau$ at the estimated time of transmission $t_{\text {trans }}$. We estimated $t_{\text {trans }}$ to be approximately 30 days prior to collection of the first recipient sample (day 0 ) for $e n v$ and 40 days for gag (Table 2 ). We calculated $N_{R} \tau\left(t_{\text {trans }}\right)$ to be 1.6 for $e n v V 1-V 4$, and 2.0 for gag p24 (Table 2). These values are near the lower prior boundary of one and their posterior distributions both exhibit a large positive skew (Figure 3 ). The level of diversity in the donor at the time of transmission $N_{D} \tau$ was compared with that which was transmitted $N_{R} \tau\left(t_{\text {trans }}\right)$ as a percentage ratio $\delta$. For $e n v, N_{D} \tau$ was estimated to be 1014 , giving a value of $\delta$ as $0.17 \%$. For $g a g$ p $24, N_{D} \tau$ was 771 , giving $\delta$ as $0.29 \%$ (Table 2).

Importantly, if selection was acting on $e n v$ to restrict the proportion of variants capable of establishing a new infection, we would expect a greater loss of diversity in this region when compared to gag, assuming recombination between the two regions. Therefore, the similarity in $\delta$ between $e n v$ and gag argues against strong selection at transmission.

We conclude that $>99 \%$ of genetic diversity in the donor viral population, in both $e n v$ and gag, was lost during this case of horizontal transmission. A reduction in viral diversity after horizontal transmission has been reported fre- quently in the literature [1,3-6]. However, information regarding the diversity present in the donor is often lacking, and even in cases where this data exists $[2,7]$ it is difficult to measure levels of diversity close to the transmission event. The method implemented here overcomes this problem, estimating genetic diversity at the inferred time of transmission, and therefore allows accurate quantification of the transmission bottleneck.

To generalise this result we next investigated diversity $(N \tau)$ of the founding viral population in nine patients infected through homosexual contact for which donor sequences were unavailable. Sequences had been published previously [29]. Assuming the best-fit demographic model, $N \tau$ at seroconversion was found to vary between around 1720 and 8 (mean: 406; Table 3). In the recipient of the transmitter pair, $N \tau$ at seroconversion (day 0 ) was 1150 (HPD upper: 1930), which is not significantly different ( $p=0.302$; one-sample $t$-test).

Finally, to compare the diversity present close to the time of infection in patients infected via two different modes of transmission, we estimated $N \tau$ at birth (transmission) in 27 vertically infected infants. The average $N \tau$ at birth was 696 (Table 3). Although we were unable to detect a bottleneck at transmission in eight of the infants (p2, p3, p6, p8, $\mathrm{pa}, \mathrm{pd}, \mathrm{pc}$ and $\mathrm{pd}$ ), the estimates for $N \tau$ close to the time of infection in the horizontally and vertically infected patient groups were not significantly different $(p=0.320$; two-sample $t$-test). 


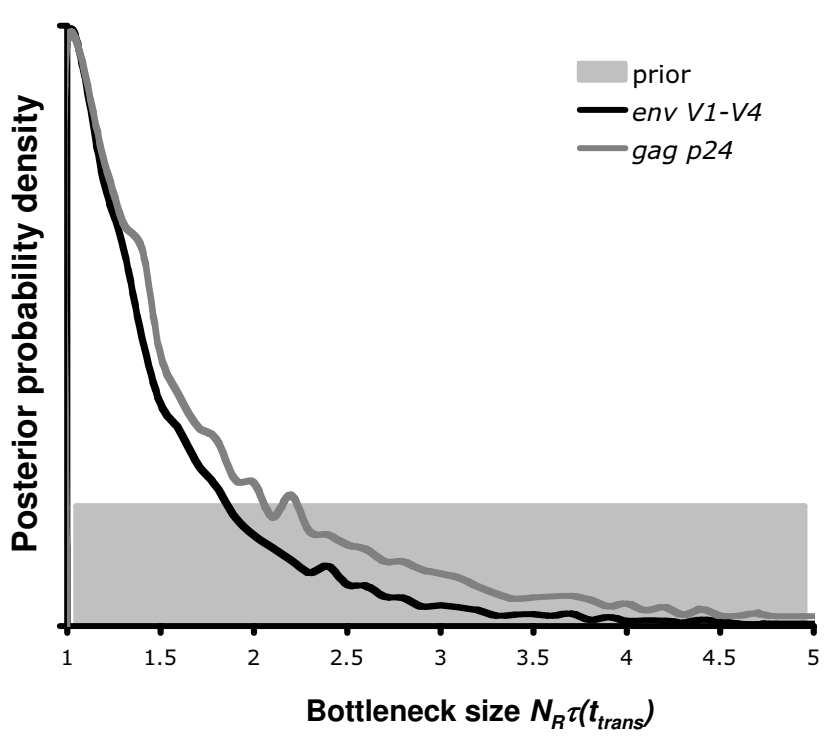

Figure 3

Effective population size at transmission $\mathbf{N}_{R} \tau\left(t_{\text {trans }}\right)$. The marginal posterior probability density of $N_{R} \tau\left(t_{\text {trans }}\right)$ is shown for both env VI-V4 and gag p24. The shaded area represents the uniform prior distribution that was used, with a minimum bound of one.

\section{Discussion}

From our analysis of a single donor and recipient transmission pair, we conclude that in this case the viral diversity sampled during homosexual transmission of HIV-1 was very small $(<1 \%)$. This result was consistent for both $e n v$ and gag. Interpretation of our finding is dependent on whether transmission is considered a neutral or selective process. In particular, if transmission is neutral it can be concluded from the severe bottleneck reported here that the consequent genetic drift will be strong, with negative consequences for viral fitness. Natural selection on the other hand is likely to mitigate any deleterious effects of genetic drift associated with transmission.

It is possible that the diversity present in the inoculum itself was larger, and that selection acting on $e n v$ restricted propagation of the new infection to a few members of the initial population [30]. The similar levels of diversity observed in env and gag could then be explained by genetic coupling between the two regions. The frequency with which recombination occurs in HIV-1 [14-16] argues against such linkage, suggesting that independent selective forces acting on env and gag must be invoked to explain this observation. Alternatively, if transmission is neutral then our estimate of the diversity transmitted will be closer to the diversity actually present in the inoculum. This will have implications for the replicative fitness of the viral population responsible for founding a new infection.
Indeed, it has been shown experimentally that a random population bottleneck of a single clone can have severe consequences for the replicative fitness of HIV-1 [21]. Furthermore, by lowering their chances of transmission, genetic drift has the potential to prevent the accumulation of advantageous changes at the population level, thereby impeding the long-term adaptation of HIV-1 [31].

Neutral transmission also means that the degree of genetic diversity passed between individuals is dependent on the diversity present in the donor at the time of transmission. Because diversity in their respective donors is likely to vary greatly depending on the stage of infection [29], this could in part explain our finding of wide variation across patients in diversity of the viral population close to transmission (Table 3). Furthermore, we found the degree of variability across patients infected by the same route to be greater than any difference between groups infected via different modes of transmission (i.e. the difference between groups was not significant). Interestingly, the diversity present early in acute infection in sexually and parenterally infected individuals also appears similar [2].

We can conclude from our results that diversity of the founding population is similarly restricted during both horizontal and vertical transmission. However, it is also clear that further study is required to investigate the variability observed. For example, although a reduction in diversity is frequent [1-9], the transmission of multiple variants has also been reported during both horizontal [32] and vertical transmission [33-35], suggesting that the bottleneck is not universally restrictive.

\section{Conclusion}

Our findings quantify the contraction in genetic diversity that occurs during horizontal transmission of HIV-1. It is clear from the severity of the bottleneck that further work is required to investigate the nature of the selective forces surrounding transmission, if we are to interpret the fitness consequences for HIV-1 in the newly infected individual. Furthermore, the analyses presented suggest that the mode of transmission may not be a significant influence on the genetic diversity transmitted.

\section{Methods \\ Patient material}

The donor and recipient patients of the transmitter pair analysed here were recruited as part of an on-going study of acute HIV-1 infection and have been described in detail elsewhere [36]. The donor had been infected for at least two years prior to transmission and exhibited a stable viral load. He had not received any antiretroviral treatment. The recipient was also untreated during the time of sampling but progressed rapidly towards disease with high viral loads and low $\mathrm{CD} 4{ }^{+}$cell counts. The clinical data for 
Table 3: Estimates of viral diversity close to the time of transmission

\begin{tabular}{|c|c|c|c|c|c|}
\hline \multirow[t]{2}{*}{ Patient } & \multirow{2}{*}{$\begin{array}{l}\text { Best-fitting } \\
\text { demographic model }\end{array}$} & \multirow[t]{2}{*}{$\mu^{\mathrm{a}}$} & \multirow[t]{2}{*}{$N \tau^{\mathrm{b}}$} & \multicolumn{2}{|c|}{$N \tau$ close to transmission ${ }^{c}$} \\
\hline & & & & Meand & HPDe upper \\
\hline \multicolumn{6}{|c|}{ Horizontal transmission } \\
\hline pl & Logistic & 0.0123 & 2293 & 36.00 & 153.19 \\
\hline p2 & Logistic & 0.0166 & 4441 & 27.98 & 148.32 \\
\hline p3 & Logistic & 0.0175 & 1612 & 29.02 & 80.12 \\
\hline p5 & Exponential & 0.0223 & 2439 & 287.78 & 670.67 \\
\hline p6 & Logistic & 0.0195 & 1511 & 7.86 & 20.39 \\
\hline p7 & Logistic & 0.0085 & 8632 & 253.78 & 1173.95 \\
\hline p8 & Exponential & 0.0162 & 6003 & 1722.98 & 2911.65 \\
\hline p9 & Logistic & 0.0071 & 7168 & $|283.1|$ & 3211.35 \\
\hline pll & Logistic & 0.0128 & 6505 & 9.76 & 34.48 \\
\hline Mean & & 0.0148 & 4512 & 406.47 & 933.79 \\
\hline \multicolumn{6}{|c|}{ Vertical transmission } \\
\hline pl & Logistic & 0.0201 & 4183 & 15.48 & 79.99 \\
\hline p2 & Constant & 0.0560 & 275 & 275.46 & 511.97 \\
\hline p3 & Constant & 0.0163 & 383 & 383.27 & 714.69 \\
\hline p4 & Exponential & 0.0098 & 67696 & 1214.76 & 5810.63 \\
\hline p5 & Exponential & 0.0133 & 7372 & 1360.46 & 3444.98 \\
\hline p6 & Constant & 0.0251 & 183 & 181.98 & 323.10 \\
\hline p7 & Exponential & 0.0226 & 1165 & 151.26 & 294.62 \\
\hline p8 & Constant & 0.0145 & 521 & 521.84 & 857.01 \\
\hline p9 & Exponential & 0.0120 & 2740 & 191.34 & 405.22 \\
\hline plo & Logistic & 0.0188 & 1050 & 384.47 & 877.21 \\
\hline pll & Logistic & 0.0163 & 740 & $4 \mid 0.67$ & 926.82 \\
\hline $\mathrm{pl} 2$ & Exponential & 0.0206 & 1730 & 121.83 & 254.40 \\
\hline pl3 & Exponential & 0.0218 & 2603 & 81.27 & 173.89 \\
\hline pl4 & Exponential & 0.0164 & 1865 & 208.00 & 411.70 \\
\hline pl5 & Logistic & 0.0397 & 889 & 1.55 & 4.80 \\
\hline p16 & Logistic & 0.0173 & 269904 & 261.90 & 577.67 \\
\hline pl8 & Logistic & 0.0097 & 146723 & 960.34 & 1978.63 \\
\hline pla & Exponential & 0.0095 & 2842 & 342.42 & 655.38 \\
\hline p2I & Exponential & 0.0053 & 302840 & 1712.90 & 4936.78 \\
\hline p22 & Exponential & 0.0046 & 547670 & 3159.60 & 11050.00 \\
\hline p23 & Logistic & 0.0093 & 123360 & 371.85 & 706.09 \\
\hline p24 & Logistic & 0.0102 & 97018 & 524.69 & 814.59 \\
\hline p25 & Logistic & 0.0071 & 2508 & 2194.63 & 4321.04 \\
\hline $\mathrm{pa}$ & Constant & 0.0280 & 640 & 638.53 & 895.76 \\
\hline $\mathrm{pb}$ & Constant & 0.0076 & 2006 & 2000.48 & 3593.83 \\
\hline $\mathrm{pc}$ & Constant & 0.0094 & 879 & 879.45 & 1626.67 \\
\hline pd & Constant & 0.0146 & 254 & 254.26 & 526.95 \\
\hline Mean & & 0.0169 & 58890 & 696.47 & 1732.39 \\
\hline
\end{tabular}

aSubstitution rate in number of changes per site per year

bProduct of the effective population size and generation time in days at the most recent time point

cSeroconversion or birth for horizontally and vertically infected patients respectively

dMean of the marginal posterior probability distribution of parameter values

eHighest Posterior Density

both donor and recipient during sampling is given in Additional file 1.

The first recipient sample (day 0) was collected six weeks after he last tested PCR (polymerase chain reaction) nega- tive for HIV-1 DNA and RNA. Three additional samples from the recipient were available at days 11,59 and 237. Donor samples were collected 70 and 155 days prior to the first sample from the recipient. Gag p24 (834bp) and the V1-V4 region of the env gene ( $951 \mathrm{bp}$ ) were sequenced 
from viral RNA. Envelope sequences were obtained from all time points using previously described methods [37], yielding a total of 100 clones (average: 17 clones per time point; range: 12-21). For gag p24, a total of 100 clones were sequenced from all time points except day 0 (average: 20 clones per time point; range: 10-28). Details of viral loads from each time point are listed in Additional file 1 . Sequences are available from GenBank under accession numbers DQ316399-DQ316601.

Envelope sequences were also obtained from 27 HIV-1 positive children. All were HIV negative by PCR at birth indicating that infection occurred peri-partum rather than in utero. Detailed descriptions of the cohort $[38,39]$ and sequencing techniques [40] are given elsewhere. The clinical prognosis of each patient is given in Additional file 2. Sequences were around 360bp in length, spanning the highly variable envelope V3 region. Multiple clones were collected from serial time points post-infection (Additional file 2). All sequences (excepting those from $\mathrm{pa}, \mathrm{pb}$, pc and pd) were derived from viral RNA. These sequences are available from GenBank under accession numbers $\underline{\text { AY823998 }}$ - $\underline{\text { AY824946. }}$

\section{Phylogenetic inference}

Sequences were first aligned manually using Se-Al [41]. Maximum likelihood phylogenies for env V1-V4 and gag p24 sequences were then constructed using PAUP* [42]. Estimation assumed the HKY85 $+\mathrm{I}+\mathrm{d} \Gamma_{4}$ model of nucleotide substitution $[43,44]$. All parameters were inferred from the data using maximum likelihood.

\section{Quantification of the diversity lost during horizontal transmission}

Within a coalescent framework, and assuming the HKY85 $+\mathrm{d} \Gamma_{4}$ model of nucleotide substitution [43,44], four demographic models were fitted to the transmission pair sequence data.

$$
\begin{aligned}
& \text { Null model: } N_{t}=N_{R}=N_{D} \quad[1] \\
& \text { Constant-Constant: } N_{t}= \begin{cases}N_{R} & t \leq t_{\text {trans }} \\
N_{D} & t>t_{\text {trans }}\end{cases} \\
& \text { Exponential-Constant : } N_{t}=\left\{\begin{array}{cc}
N_{R} e^{-r t} & t \leq t_{\text {trans }} \\
N_{D} & t>t_{\text {trans }}
\end{array}\right. \\
& \text { Logistic-Constant: } \quad N_{t}=\left\{\begin{array}{cc}
\frac{N_{R}(1+c) e^{-r t}}{c+e^{-r t}} & t \leq t_{\text {trans }} \\
N_{D} & t>t_{\text {trans }}
\end{array}\right.
\end{aligned}
$$

where $c=\frac{1}{e^{r t_{50}}-2}$
All substitution and demographic parameters, including the time of transmission $t_{\text {trans }}$ growth rate $r$, and mid-time of the population $t_{50}$, were estimated from the data within a Bayesian coalescent framework by Markov chain Monte Carlo (MCMC), using the BEAST program [45]. Bayesian MCMC estimates each parameter as the mean of its marginal posterior probability distribution, whilst simultaneously incorporating uncertainty in the underlying genealogy and other parameters. Diversity of the viral population is given as the product of the effective population size and generation length in days $N \tau$ [27].

Uncertainty in the estimated parameter values is summarized by the highest posterior density (HPD) interval, which contains $95 \%$ of the marginal posterior distribution. The length of the MCMC chain was chosen so that the effective sample size (ESS) for each parameter was > 100 , indicating that parameter space had been sufficiently explored [24]. Since it consistently gave the lowest value, the coalescent ESS (the number of effectively independent log likelihoods sampled from the coalescent posterior distribution) for each model is given in Table 1. All priors were assumed to be uniform on a natural scale, including the effective population size in the recipient at transmis$\operatorname{sion} N_{R} \tau\left(t_{\text {trans }}\right)$. The prior boundaries for the time of transmission $t_{\text {trans }}$ were set from when the recipient was last confirmed HIV-1 negative ( 53 days before the first recipient sample) to the time at which the first recipient sample was collected (day 0 ). We placed a minimum prior bound of one on $N_{R} \tau\left(t_{\text {trans }}\right)$. With the exception of $t_{\text {trans }}$ and $N_{R} \tau\left(t_{\text {trans }}\right)$, the MCMC chain did not impinge on any of the prescribed prior boundaries for the models tested.

The relative fit of each model to the data was assessed using the Akaike Information Criteria (AIC) [46]. The AIC of a given model is twice its marginal log likelihood plus the number of parameters specified (AIC $=2 l n L k+2 p)$. The model with the lowest AIC is selected as the best representation of the data.

Selection of the appropriate demographic model allowed us to calculate $N_{R} \tau\left(t_{\text {trans }}\right)$ and quantify the amount of diversity lost at transmission through a comparison of $N_{R} \tau\left(t_{\text {trans }}\right)$ with $N_{D} \tau$ as the percentage ratio $\delta$.

\section{Bayesian skyline plot}

The skyline plot is a piecewise-constant model of population size that estimates $N \tau$ for each coalescent interval of the genealogy $[47,48]$. It allows the demographic history of a population to be reconstructed without $a$ priori specification of a particular model. The Bayesian skyline extends the generalised skyline plot [48] to take into account serial sequence sampling times and an uncertain genealogy [28]. The distribution of skyline plots is sampled using MCMC according to their posterior probabili- 
ties given the sequence data, to produce an estimate and HPD confidence intervals of the effective population size through time. The Bayesian skyline plot was estimated using BEAST [45], allowing ten steps in $N \tau$ through time.

\section{Authors' contributions}

CTTE collected the data, performed the analysis and wrote the paper. ECH contributed to the design of the study and the writing of the manuscript. DJW assisted with the analysis. RPV and EJA collected sequences for the infant data set. REP contributed to the design of the study and provided the funding. AJD contributed to the design of the study, the development of the software, the analysis and the writing of the article.

\section{Additional material}

\section{Additional File 1}

Clinical data for transmission pair

Click here for file

[http://www.biomedcentral.com/content/supplementary/1471-

2148-6-28-S1.doc]

\section{Additional File 2}

Clinical categorisation and sequencing profile of vertically infected infants Click here for file

[http://www.biomedcentral.com/content/supplementary/14712148-6-28-S2.doc]

\section{Acknowledgements}

This work was supported by the Wellcome Trust (CTTE, AJD, ECH and REP) and Biotechnology and Biological Sciences Research Council (DJW).

\section{References}

I. Delwart E, Magierowska M, Royz M, Foley B, Peddada L, Smith R, Heldebrant C, Conrad A, Busch M: Homogeneous quasispecies in 16 out of 17 individuals during very early HIV-I primary infection. Aids 2002, 16:189-195.

2. Wolfs TF, Zwart G, Bakker M, Goudsmit J: HIV-I genomic RNA diversification following sexual and parenteral virus transmission. Virology 1992, I89: I03-II0.

3. McNearney T, Hornickova Z, Markham R, Birdwell A, Arens M, Saah $A$, Ratner $L$ : Relationship of human immunodeficiency virus type I sequence heterogeneity to stage of disease. Proc Natl Acad Sci U S A 1992, 89: 10247-10251.

4. Pang S, Shlesinger Y, Daar ES, Moudgil T, Ho DD, Chen IS: Rapid generation of sequence variation during primary HIV-I infection. Aids 1992, 6:453-460.

5. Cichutek K, Merget H, Norley S, Linde R, Kreuz W, Gahr M, Kurth $\mathrm{R}$ : Development of a quasispecies of human immunodeficiency virus type I in vivo. Proc Natl Acad Sci U S A I992, 89:7365-7369.

6. Zhang LQ, MacKenzie P, Cleland A, Holmes EC, Brown AJ, Simmonds $P$ : Selection for specific sequences in the external envelope protein of human immunodeficiency virus type I upon primary infection. J Virol 1993, 67:3345-3356.

7. Zhu T, Mo H, Wang N, Nam DS, Cao Y, Koup RA, Ho DD: Genotypic and phenotypic characterization of HIV-I patients with primary infection. Science 1993, 26 I:II79-II8I.

8. Ahmad N, Baroudy BM, Baker RC, Chappey C: Genetic analysis of human immunodeficiency virus type I envelope $V 3$ region isolates from mothers and infants after perinatal transmission. J Virol 1995, 69:1001-1012.

9. Wolinsky SM, Wike CM, Korber BT, Hutto C, Parks WP, Rosenblum LL, Kunstman KJ, Furtado MR, Munoz JL: Selective transmission of human immunodeficiency virus type-I variants from mothers to infants. Science 1992, 255: II34-1 I37.

10. Domingo E, Sabo D, Taniguchi T, Weissmann C: Nucleotide sequence heterogeneity of an RNA phage population. Cell 1978, 13:735-744.

II. Duarte EA, Novella IS, Ledesma S, Clarke DK, Moya A, Elena SF, Domingo E, Holland J]: Subclonal components of consensus fitness in an RNA virus clone. I Virol 1994, 68:4295-430 I.

12. Sanjuan R, Moya A, Elena SF: The distribution of fitness effects caused by single-nucleotide substitutions in an RNA virus. Proc Natl Acad Sci U S A 2004, I 0 I:8396-840I.

13. Muller HJ: The Relation of Recombination to Mutational Advance. Mutat Res 1964, 106:2-9.

14. Jung A, Maier R, Vartanian JP, Bocharov G, Jung V, Fischer U, Meese E, Wain-Hobson S, Meyerhans A: Recombination: Multiply infected spleen cells in HIV patients. Nature 2002, 4I 8: I 44.

I5. Zhuang J, Jetzt AE, Sun G, Yu H, Klarmann G, Ron Y, Preston BD, Dougherty JP: Human immunodeficiency virus type I recombination: rate, fidelity, and putative hot spots. I Virol 2002 , 76: I I273-I I 282

16. Rhodes T, Wargo H, Hu WS: High rates of human immunodeficiency virus type I recombination: near-random segregation of markers one kilobase apart in one round of viral replication. J Virol 2003, 77: I I193-I1200.

17. Chao L: Fitness of RNA virus decreased by Muller's ratchet. Nature 1990, 348:454-455.

18. Escarmis C, Davila M, Charpentier N, Bracho A, Moya A, Domingo E: Genetic lesions associated with Muller's ratchet in an RNA virus. J Mol Biol 1996, 264:255-267.

19. Duarte E, Clarke D, Moya A, Domingo E, Holland J: Rapid fitness losses in mammalian RNA virus clones due to Muller's ratchet. Proc Natl Acad Sci U S A 1992, 89:6015-60I9.

20. Clarke DK, Duarte EA, Moya A, Elena SF, Domingo E, Holland J: Genetic bottlenecks and population passages cause profound fitness differences in RNA viruses. J Virol 1993, 67:222-228.

21. Yuste E, Sanchez-Palomino S, Casado C, Domingo E, Lopez-Galindez $C$ : Drastic fitness loss in human immunodeficiency virus type I upon serial bottleneck events. J Virol |999, 73:2745-275 I.

22. Novella IS, Elena SF, Moya A, Domingo E, Holland J]: Size of genetic bottlenecks leading to virus fitness loss is determined by mean initial population fitness. J Virol 1995, 69:2869-2872.

23. Bergstrom CT, McElhany P, Real LA: Transmission bottlenecks as determinants of virulence in rapidly evolving pathogens. Proc Natl Acad Sci U S A 1999, 96:5095-5100.

24. Drummond AJ, Nicholls GK, Rodrigo AG, Solomon W: Estimating mutation parameters, population history and genealogy simultaneously from temporally spaced sequence data. Genetics 2002, 16 I:1307-1320.

25. Pybus OG, Drummond AJ, Nakano T, Robertson BH, Rambaut A: The epidemiology and iatrogenic transmission of hepatitis $C$ virus in Egypt: a Bayesian coalescent approach. Mol Biol Evol 2003, 20:38l-387.

26. Shapiro B, Drummond AJ, Rambaut A, Wilson MC, Matheus PE, Sher AV, Pybus OG, Gilbert MT, Barnes I, Binladen J, Willerslev E, Hansen AJ, Baryshnikov GF, Burns JA, Davydov S, Driver JC, Froese DG, Harington CR, Keddie G, Kosintsev P, Kunz ML, Martin LD, Stephenson RO, Storer J, Tedford R, Zimov S, Cooper A: Rise and fall of the Beringian steppe bison. Science 2004, 306:|56|-I 565.

27. Rodrigo AG, Felsenstein J: Coalescent approaches to HIV population genetics. In The Evolution of HIV Edited by: Crandall KA. Baltimore, The Johns Hopkins University Press; 1999:233-272.

28. Drummond AJ, Rambaut A, Shapiro B, Pybus OG: Bayesian Coalescent Inference of Past Population Dynamics from Molecular Sequences. Mol Biol Evol 2005, In press:.

29. Shankarappa R, Margolick JB, Gange SJ, Rodrigo AG, Upchurch D, Farzadegan H, Gupta P, Rinaldo CR, Learn GH, He X, Huang XL, Mullins Il: Consistent Viral Evolutionary Changes Associated with the Progression of Human Immunodeficiency Virus Type I Infection. J Virol 1999, 73:10489-10502.

30. Learn GH, Muthui D, Brodie SJ, Zhu T, Diem K, Mullins Jl, Corey L: Virus population homogenization following acute human 
immunodeficiency virus type I infection. I Virol 2002, 76:11953-11959.

31. Rambaut A, Posada D, Crandall KA, Holmes EC: The causes and consequences of HIV evolution. Nat Rev Genet 2004, 5:52-6I.

32. Long EM, Martin HLJ, Kreiss JK, Rainwater SM, Lavreys L, Jackson DJ, Rakwar J, Mandaliya K, Overbaugh J: Gender differences in HIV-I diversity at time of infection. Nat Med 2000, 6:7I-75.

33. Pasquier C, Cayrou C, Blancher A, Tourne-Petheil C, Berrebi A, Tricoire J, Puel J, lzopet J: Molecular evidence for mother-to-child transmission of multiple variants by analysis of RNA and DNA sequences of human immunodeficiency virus type $I$. J Virol 1998, 72:8493-850I.

34. Wade CM, Lobidel D, Brown AJ: Analysis of human immunodeficiency virus type $I$ env and gag sequence variants derived from a mother and two vertically infected children provides evidence for the transmission of multiple sequence variants. J Gen Virol 1998, 79 ( Pt 5): 1055-1068.

35. Dickover RE, Garratty EM, Plaeger S, Bryson YJ: Perinatal transmission of major, minor, and multiple maternal human immunodeficiency virus type I variants in utero and intrapartum. J Virol 200I, 75:2194-2203.

36. Milicic A, Edwards CTT, Hue S, Fox J, Brown H, Pillay T, Drijfhout JW, Weber JN, Holmes EC, Fidler SJ, Zhang HT, Phillips RE: Sexual transmission of single HIV-I virions encoding highly polymorphic multi-site CTL escape variants. J Virol 2005, 79:13953-13962.

37. Oxenius A, Price DA, Trkola A, Edwards C, Gostick E, Zhang HT, Easterbrook PJ, Tun T, Johnson A, Waters A, Holmes EC, Phillips RE: Loss of viral control in early HIV-I infection is temporally associated with sequential escape from CD8+ T cell responses and decrease in HIV-I-specific CD4+ and CD8+ T cell frequencies. J Infect Dis 2004, 190:713-72I.

38. Abrams EJ, Matheson PB, Thomas PA, Thea DM, Krasinski K, Lambert G, Shaffer N, Bamji M, Hutson D, Grimm K, et al.: Neonatal predictors of infection status and early death among 332 infants at risk of HIV-I infection monitored prospectively from birth. New York City Perinatal HIV Transmission Collaborative Study Group. Pediatrics 1995, 96:45I-458.

39. Thomas PA, Weedon J, Krasinski K, Abrams E, Shaffer N, Matheson P, Bamji M, Kaul A, Hutson D, Grimm KT, et al.: Maternal predictors of perinatal human immunodeficiency virus transmission. The New York City Perinatal HIV Transmission Collaborative Study Group. Pediatr Infect Dis J 1994, 13:489-495.

40. Strunnikova N, Ray SC, Livingston RA, Rubalcaba E, Viscidi RP: Convergent evolution within the V3 loop domain of human immunodeficiency virus type $I$ in association with disease progression. J Virol 1995, 69:7548-7558.

4I. Rambaut A: Se-Al. Sequence Alignment Editor v2.0a II. 2002 [http://evolve.zoo.ox.ac.uk].

42. Swofford R: PAUP*. Phylogenetic Analysis Using Parsimony (* and other methods). Version 4 edition. , Sinauer Associates, Sunderland, Massachusetts; 2002.

43. Hasegawa $M$, Kishino $H$, Yano $T$ : Dating of the human-ape splitting by a molecular clock of mitochondrial DNA. J Mol Evol 1985, 22:160-174.

44. Yang Z: Maximum likelihood phylogenetic estimation from DNA sequences with variable rates over sites: approximate methods. J Mol Evol 1994, 39:306-314.

45. Drummond A, Rambaut A: BEAST. Bayesian Evolutionary Analysis Sampling Trees vI.I. I.I2004 [http://evolve.zoo.ox.ac.uk/ beast $/]$.

46. Akaike $\mathrm{H}$ : Information theory as an extension of the maximum likelihood principle: ; Akademiai Kiado, Budapest. Edited by: Petrov BN and Csaki F. ; 1973:267-28I.

47. Pybus OG, Rambaut $A$, Harvey PH: An integrated framework for the inference of viral population history from reconstructed genealogies. Genetics 2000, I55:1429-I 437.

48. Strimmer K, Pybus OG: Exploring the demographic history of DNA sequences using the generalized skyline plot. Mol Biol Evol 200I, I 8:2298-2305.
Publish with Bio Med Central and every scientist can read your work free of charge

"BioMed Central will be the most significant development for disseminating the results of biomedical research in our lifetime. "

Sir Paul Nurse, Cancer Research UK

Your research papers will be:

- available free of charge to the entire biomedical community

- peer reviewed and published immediately upon acceptance

- cited in PubMed and archived on PubMed Central

- yours - you keep the copyright

Submit your manuscript here:

http://www.biomedcentral.com/info/publishing_adv.asp
BiolMedcentral 